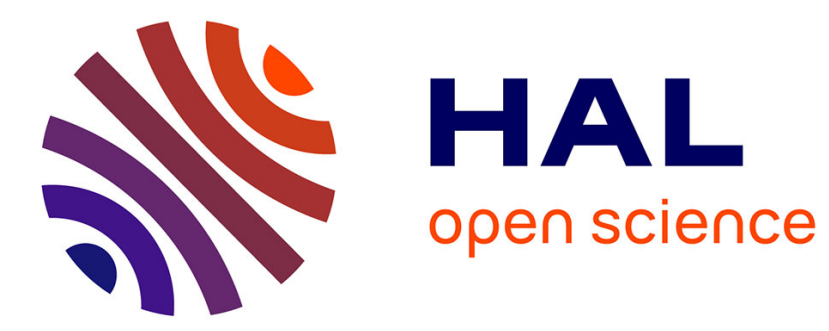

\title{
On a sum involving the Euler totient function
}

Jie $\mathrm{Wu}$

\section{- To cite this version:}

Jie $\mathrm{Wu}$. On a sum involving the Euler totient function. Indagationes Mathematicae, 2019, 30 (4), pp.536-541. 10.1016/j.indag.2019.01.009 . hal-01884018v2

\section{HAL Id: hal-01884018 \\ https://hal.science/hal-01884018v2}

Submitted on 3 Oct 2018

HAL is a multi-disciplinary open access archive for the deposit and dissemination of scientific research documents, whether they are published or not. The documents may come from teaching and research institutions in France or abroad, or from public or private research centers.
L'archive ouverte pluridisciplinaire HAL, est destinée au dépôt et à la diffusion de documents scientifiques de niveau recherche, publiés ou non, émanant des établissements d'enseignement et de recherche français ou étrangers, des laboratoires publics ou privés. 


\title{
ON A SUM INVOLVING THE EULER TOTIENT FUNCTION
}

\author{
J. WU
}

AbSTRACT. In this short note, we prove that

$$
\frac{4}{\pi^{2}} x \log x+O(x) \leqslant \sum_{n \leqslant x} \varphi\left(\left[\frac{x}{n}\right]\right) \leqslant\left(\frac{1}{3}+\frac{4}{\pi^{2}}\right) x \log x+O(x),
$$

for $x \rightarrow \infty$, where $\varphi(n)$ is the Euler totient function and $[t]$ is the integral part of real $t$.

This improves recent results of Bordellès-Heyman-Shparlinski and of Dai-Pan.

\section{INTRODUCTION}

As usual, denote by $\varphi(n)$ the Euler totient function and by $[t]$ the integral part of real $t$. Very recently, Bordellès, Heyman and Shparlinski [1] studied the asymptotic behaviour of the summatory function

$$
S(x):=\sum_{n \leqslant x} \varphi\left(\left[\frac{x}{n}\right]\right)
$$

and proved that

$$
\begin{aligned}
& S(x) \leqslant\left(\frac{1}{2}+\frac{3}{\pi^{2}}\right) x \log x+4 x+\frac{\sqrt{x} \log x}{4}+\sqrt{x} \quad(x \geqslant 3), \\
& S(x) \geqslant\left(\frac{2629}{4009} \cdot \frac{6}{\pi^{2}}+o(1)\right) x \log x \quad(x \rightarrow \infty) .
\end{aligned}
$$

They also posed a question : Is it true that

$$
S(x)=\left(\frac{6}{\pi^{2}}+o(1)\right) x \log x
$$

as $x \rightarrow \infty$ ? Some numerical evidences were given.

The aim of this short note is to improve Bordellès, Heyman and Shparlinski's (1.2) and (1.3), by refining their method. Our result is as follows.

Theorem 1. We have

$$
\frac{4}{\pi^{2}} x \log x+O(x) \leqslant S(x) \leqslant\left(\frac{1}{3}+\frac{4}{\pi^{2}}\right) x \log x+O(x)
$$

for $x \rightarrow \infty$.

Date: October 2, 2018.

2010 Mathematics Subject Classification. 11N36, 11L05.

Key words and phrases. Euler totient function, exponential sums. 
For comparaison, we have

$$
\left\{\begin{array} { l } 
{ \frac { 1 } { 2 } + \frac { 3 } { \pi ^ { 2 } } \approx 0 . 8 0 3 9 6 , } \\
{ \frac { 2 6 2 9 } { 4 0 0 9 } \frac { 6 } { \pi ^ { 2 } } \approx 0 . 3 9 8 4 1 , }
\end{array} \text { and } \quad \left\{\begin{array}{l}
\frac{1}{3}+\frac{4}{\pi^{2}} \approx 0.73861, \\
\frac{4}{\pi^{2}} \approx 0.40528 .
\end{array}\right.\right.
$$

As indicated by Bordellès, Heyman and Shparlinski in [1], the proof of (1.2) is rather elementary. But the lower bound (1.3) uses a much deeper approach relying on the theory of exponential pairs. In particular, to obtain the numerically stronger result, they used the recently discovered exponent pair $\left(\frac{13}{84}+\varepsilon, \frac{55}{84}+\varepsilon\right)$ by Bourgain [2]. Let $\psi(t):=t-[t]-\frac{1}{2}$ and $\delta \in\{0,1\}$. For $x \geqslant 2$ and $1 \leqslant D \leqslant x$, define

$$
\mathfrak{S}_{\delta}(x, D):=\sum_{D<d \leqslant 2 D} \varphi(d) \psi\left(\frac{x}{d+\delta}\right) .
$$

The key of Bordellès, Heyman and Shparlinski's method is to find $\theta$ as large as possible such that

$$
\mathfrak{S}_{\delta}\left(x, x^{\theta}\right) \ll x .
$$

They shew that $\theta=\frac{2629}{4009} \approx 0.65577$ is admissible and obtained the constant $\theta \frac{6}{\pi^{2}}$ in the lower bound (1.3).

Our improvements come from two simple observations:

(a) Firstly we give a more careful treatment for the related exponential sum (see Proposition 2.1 below). This allows us to show that $\theta=\frac{2}{3} \approx 0.66666$ is admissible for (1.6) and to get a better lower. It is worth to note that our argument is simpler and that we do not need to use Bourgain's new exponent pair. In fact we only use the simplest exponent pair $\left(\frac{1}{2}, \frac{1}{2}\right)$, i.e. van der Corput inequality.

(b) Our argument about upper bound part is different from [1]. In order to improve upper bound (1.2), we introduced exponential sum technique to show that the constant in the upper bound can be improved to $1-\theta+\theta \frac{6}{\pi^{2}}$.

It should be possible to further improve slightly the constants in Theorem 1 by applying combinatorial identity on the Möbius function and more sophistic methods of multiple exponential sums (see $[4,6]$ ).

When we redact our manuscript, we note that Lixia Dai \& Hao Pan [3] also have

remarked (b) above and obtained a better upper bound constant $\frac{1380}{4009}+\frac{2629}{4009} \frac{6}{\pi^{2}} \approx 0.74288$ than (1.2) by using Bordellès, Heyman and Shparlinski's $\theta=\frac{2629}{4009}$ value.

\section{A Key estimate}

The aim of this section is to prove the following bound for $\mathfrak{S}_{\delta}(x, D)$, which will play a key role in proof of Theorem 1.

Proposition 2.1. Under the previous notation, we have

$$
\mathfrak{S}_{\delta}(x, D) \ll\left(x^{\kappa} D^{1+\lambda}\right)^{1 /(\kappa+1)}+x^{\kappa} D^{-2 \kappa+\lambda} \log x+x^{-1} D^{3},
$$

where $(\kappa, \lambda)$ is an exponent pair. 


\subsection{Two preliminary lemmas.}

In order prove Proposition 2.1, we need two standard lemmas in the exponential sum theory. The first one is due to Vaaler (see [5, Theorem A.6] or [1, Lemma 4.3]).

Lemma 2.2. For $x \geqslant 1$ and $H \geqslant 1$, we have

$$
\psi(x)=-\sum_{1 \leqslant|h| \leqslant H} \Phi\left(\frac{h}{H+1}\right) \frac{\mathrm{e}(h x)}{2 \pi \mathrm{i}}+R_{H}(x),
$$

where $\Phi(t):=\pi t(1-|t|) \cot (\pi t)+|t|$ and the error term $R_{H}(x)$ satisfies

$$
\left|R_{H}(x)\right| \leqslant \frac{1}{2 H+2} \sum_{0 \leqslant|h| \leqslant H}\left(1-\frac{|h|}{H+1}\right) \mathrm{e}(h x) .
$$

The second lemma is [5, Lemma 2.4].

Lemma 2.3. Let $L(Q):=\sum_{j=1}^{J} C_{j} Q^{c_{j}}+\sum_{k=1}^{K} D_{k} Q^{-d_{k}}$, where $C_{j}, c_{j}, D_{k}, d_{k}>0$. For any $Q \geqslant Q^{\prime}>0$, there exists some $Q_{1} \in\left[Q^{\prime}, Q\right]$ such that

$$
L\left(Q_{1}\right) \ll \sum_{j=1}^{J} \sum_{k=1}^{K}\left(C_{j}^{d_{k}} D_{k}^{c_{j}}\right)^{1 /\left(c_{j}+d_{k}\right)}+\sum_{j=1}^{J} C_{j} Q^{\prime c_{j}}+\sum_{k=1}^{K} D_{k} Q^{-d_{k}} .
$$

\subsection{Proof of Proposition 2.1.}

As usual, denote by $\mu(n)$ the Möbius function. By using the relation

$$
\varphi(d)=\sum_{m n=d} \mu(m) n
$$

and by a simple partial summation, we can write

$$
\begin{aligned}
\mathfrak{S}_{\delta}(x, D) & =\sum_{m \leqslant 2 D} \mu(m) \sum_{D / m<n \leqslant 2 D / m} n \psi\left(\frac{x}{m n+\delta}\right) . \\
& \ll \sum_{m \leqslant 2 D} \frac{D}{m} \max _{D / m<N \leqslant 2 D / m}\left|\mathfrak{S}_{\delta}(x, D, m, N)\right|,
\end{aligned}
$$

where

$$
\mathfrak{S}_{\delta}(x, D, m, N):=\sum_{D / m<n \leqslant N} \psi\left(\frac{x}{m n+\delta}\right) .
$$

With the help of Lemma 2.3 and noticing the fact that $0<\Phi(t)<1(0<|t|<1)$, we can derive

$$
\mathfrak{S}_{\delta}(x, D, m, N) \ll \frac{N}{H}+\sum_{h \leqslant H} \frac{1}{h}\left|\sum_{D / m<n \leqslant N} \mathrm{e}\left(\frac{h x}{m n+\delta}\right)\right|
$$

for $D / m<N \leqslant 2 D / m$ and $1 \leqslant H \leqslant N$.

Applying the exponent pair $(\kappa, \lambda)$ to the inner sum over $n$, we find that

$$
\begin{aligned}
\mathfrak{S}_{\delta}(x, D, m, N) & \ll \frac{N}{H}+\sum_{h \leqslant H} \frac{1}{h}\left\{\left(\frac{h x}{m N^{2}}\right)^{\kappa} N^{\lambda}+\frac{m N^{2}}{h x}\right\} \\
& \ll H^{-1} N+x^{\kappa} H^{\kappa} m^{-\kappa} N^{-2 \kappa+\lambda}+x^{-1} m N^{2}
\end{aligned}
$$


for all $H \in[1, N]$. According to Lemma 2.3, there is a $H \in[1, N]$ such that

$$
\begin{aligned}
\mathfrak{S}_{\delta}(x, D, m, N) & \ll\left(x^{\kappa} m^{-\kappa} N^{-\kappa+\lambda}\right)^{1 /(\kappa+1)}+x^{\kappa} m^{-\kappa} N^{-2 \kappa+\lambda}+x^{-1} m N^{2} \\
& \ll\left(x^{\kappa} D^{-\kappa+\lambda} m^{-\lambda}\right)^{1 /(\kappa+1)}+x^{\kappa} D^{-2 \kappa+\lambda} m^{\kappa-\lambda}+x^{-1} D^{2} m^{-1} .
\end{aligned}
$$

Inserting it into (2.2), we obtain the required result.

\section{Proof of Theorem 1: Upper Bound}

Let $1 \leqslant D<\sqrt{x}$ be a parameter to be chosen later. We write

$$
S(x)=S_{1}(x)+S_{2}(x)
$$

with

$$
S_{1}(x):=\sum_{n \leqslant D} \varphi\left(\left[\frac{x}{n}\right]\right), \quad S_{2}(x):=\sum_{D<n \leqslant x} \varphi\left(\left[\frac{x}{n}\right]\right) .
$$

A. Upper bound of $S_{1}(x)$

We have trivially

$$
S_{1}(x) \leqslant \sum_{n \leqslant D} \frac{x}{n}=x \log D+O(x)
$$

B. Upper bound of $S_{2}(x)$

In order to bound $S_{2}(x)$, we put $d=[x / n]$. Then

$$
x / n-1<d \leqslant x / n \quad \text { and } \quad x /(d+1)<n \leqslant x / d .
$$

Thus

$$
\begin{aligned}
S_{2}(x) & =\sum_{d \leqslant x / D} \varphi(d) \sum_{x /(d+1)<n \leqslant x / d} 1 \\
& =\sum_{d \leqslant x / D} \varphi(d)\left(\frac{x}{d}-\psi\left(\frac{x}{d}\right)-\frac{x}{d+1}+\psi\left(\frac{x}{d+1}\right)\right) \\
& =x \sum_{d \leqslant x / D} \frac{\varphi(d)}{d(d+1)}+\sum_{d \leqslant x / D} \varphi(d)\left(\psi\left(\frac{x}{d+1}\right)-\psi\left(\frac{x}{d}\right)\right) .
\end{aligned}
$$

It is well known that

$$
\sum_{n \leqslant x} \varphi(n)=\frac{3}{\pi^{2}} x^{2}+O(x \log x), \quad \sum_{n \leqslant x} \frac{\varphi(n)}{n^{2}}=\frac{6}{\pi^{2}} \log x+O(1) .
$$

With the help of these, it follows that

$$
\sum_{d \leqslant x} \frac{\varphi(d)}{d(d+1)}=\sum_{d \leqslant x} \frac{\varphi(d)}{d^{2}}\left\{1+O\left(\frac{1}{d}\right)\right\}=\frac{6}{\pi^{2}} \log x+O(1)
$$

and

$$
\left|\sum_{d \leqslant \sqrt{x}} \varphi(d)\left(\psi\left(\frac{x}{d+1}\right)-\psi\left(\frac{x}{d}\right)\right)\right| \leqslant 2 \sum_{d \leqslant \sqrt{x}} \varphi(d) \ll x
$$


It remains to bound

$$
E(x, D):=\sum_{\sqrt{x}<d \leqslant x / D} \varphi(d)\left(\psi\left(\frac{x}{d+1}\right)-\psi\left(\frac{x}{d}\right)\right) .
$$

Let $D_{k}:=x /\left(2^{k} D\right)$ and let $K$ be the integer such that $D_{K+1}<\sqrt{x} \leqslant D_{K}$. By a simple dyadic split and by Proposition 2.1 with $(\kappa, \lambda)=\left(\frac{1}{2}, \frac{1}{2}\right)$, it follows that

$$
\begin{aligned}
|E(x, D)| & \leqslant \sum_{1 \leqslant k \leqslant K+1}\left(\left|\mathfrak{S}_{0}\left(x, D_{k}\right)\right|+\left|\mathfrak{S}_{1}\left(x, D_{k}\right)\right|\right) \\
& \ll \sum_{1 \leqslant k \leqslant K+1}\left(x^{1 / 3} D_{k}+x^{1 / 2} D_{k}^{-1 / 2} \log x+x^{-1} D_{k}^{3}\right) \\
& \ll x^{1 / 3}(x / D)+x^{1 / 2}(\log x)^{2}+x^{-1}(x / D)^{3} \\
& \ll x^{4 / 3} D^{-1}+x^{1 / 2}(\log x)^{2}+x^{2} D^{-3} .
\end{aligned}
$$

Inserting (3.4), (3.5) and (3.6) into (3.3), we find

$$
S_{2}(x) \leqslant \frac{6}{\pi^{2}} x \log (x / D)+O\left(x^{4 / 3} D^{-1}+x^{1 / 2}(\log x)^{2}+x^{2} D^{-3}\right) .
$$

C. End of the proof

Inserting (3.2) and (3.7) into (3.1) and taking $D=x^{1 / 3}$, we get the required the upper bound in (1.4).

\section{Proof of Theorem 1: Lower Bound}

Similar to (3.3), we can write

$$
S(x)=\sum_{d \leqslant x} \varphi(d) \sum_{x /(d+1)<n \leqslant x / d} 1 .
$$

Let $\sqrt{x} \leqslant D \leqslant x$ be a parameter to be chosen later. Thus

$$
\begin{aligned}
S(x) & \geqslant \sum_{d \leqslant D} \varphi(d) \sum_{x /(d+1)<n \leqslant x / d} 1 \\
& =x \sum_{d \leqslant D} \frac{\varphi(d)}{d(d+1)}+\sum_{d \leqslant D} \varphi(d)\left(\psi\left(\frac{x}{d+1}\right)-\psi\left(\frac{x}{d}\right)\right) \\
& =\frac{6}{\pi^{2}} x \log D+O(x)+R(x, D),
\end{aligned}
$$

where

$$
R(x, D):=\sum_{d \leqslant D} \varphi(d)\left(\psi\left(\frac{x}{d+1}\right)-\psi\left(\frac{x}{d}\right)\right) .
$$


Let $D_{k}:=D / 2^{k}$ and let $K$ be the integer such that $D_{K+1}<1 \leqslant D_{K}$. By a simple dyadical split and by Proposition 2.1 with $(\kappa, \lambda)=\left(\frac{1}{2}, \frac{1}{2}\right)$, it follows that

$$
\begin{aligned}
|R(x, D)| & \leqslant \sum_{1 \leqslant k \leqslant K+1}\left(\left|\mathfrak{S}_{0}\left(x, D_{k}\right)\right|+\left|\mathfrak{S}_{1}\left(x, D_{k}\right)\right|\right) \\
& \ll \sum_{1 \leqslant k \leqslant K+1}\left(x^{1 / 3} D_{k}+x^{1 / 2} D_{k}^{-1 / 2} \log x+x^{-1} D_{k}^{3}\right) \\
& \ll x^{1 / 3} D+x^{1 / 2}(\log x)^{2}+x^{-1} D^{3} .
\end{aligned}
$$

Inserting (4.2) into (4.1), we find

$$
S(x) \geqslant \frac{6}{\pi^{2}} x \log D+O\left(x^{1 / 3} D+x^{1 / 2}(\log x)^{2}+x^{-1} D^{3}\right) .
$$

The required result follows from the choice of $D=x^{2 / 3}$.

Acknowledgements. This work is supported in part by Scientific Research Innovation Team Project Affiliated to Yangtze Normal University (No. 2016XJD01). The author is thankful to Lixia Dai at Nanjing Normal University (China) for drawing his attention to Bordellès, Heyman and Shparlinski's paper [1].

\section{REFERENCES}

[1] O. Bordellès, R. Heyman \& Igor E. Shparlinski, On a sum involving the Euler function, arXiv:1808.00188v1 [math.NT] 1 Aug 2018.

[2] J. Bourgain, Decoupling, exponential sums and the Riemann zeta function, J. Amer. Math. Soc. 30 (2017), 205-224.

[3] Lixia Dai \& Hao Pan, Note on a sum involving the Euler function, arXiv:1809.10381v1 [math.NT] 27 Sep 2018.

[4] E. Fouvry and H. Iwaniec, Exponential sums with monomials, J. Number Theory 33 (1989), 311333.

[5] S. W. Graham \& G. Kolesnik, Van der Corput's Method of Exponential sums, Cambridge University Press, Cambridge, 1991.

[6] J. Wu, Almost primes in short intervals, Sci. China Math. 53 (2010), No. 9, 2511-2524.

School of Mathematics and Statistics, Yangtze Normal University, Fuling, Chongqing 200240, CHINA

Current address: CNRS LAMA 8050, Laboratoire d'Analyse et de Mathématiques Appliquées, Université Paris-Est Créteil, 94010 Créteil Cedex, France

E-mail address: jie.wu@math.cnrs.fr 